\title{
Erratum: Cuceu, C., et al. Independent Mechanisms Lead to Genomic Instability in Hodgkin Lymphoma: Microsatellite or Chromosomal Instability. Cancers 2018, 10, 233
}

\author{
Corina Cuceu ${ }^{1}$, Bruno Colicchio ${ }^{2}$, Eric Jeandidier ${ }^{3}{ }^{-}$, Steffen Junker ${ }^{4}$, François Plassa ${ }^{5}$, \\ Grace Shim ${ }^{1}$, Justyna Mika ${ }^{6}$, Monika Frenzel ${ }^{1}$, Mustafa AL Jawhari ${ }^{1}$, William M. Hempel ${ }^{1}$, \\ Sylwia Kabacik ${ }^{7}{ }^{\mathbb{D}}$, Aude Lenain ${ }^{1}$, Luc Morat ${ }^{1}$, Theodore Girinsky ${ }^{8}$, Alain Dieterlen ${ }^{2}$, \\ Joanna Polanska ${ }^{6}$ (D), Christophe Badie ${ }^{7}$, Patrice Carde ${ }^{9}$ and Radhia M'Kacher $^{1,10, *(D)}$ \\ 1 Radiobiology and Oncology Laboratory, CEA, iRCM, 92265 Fontenay aux Roses CEDEX, France; \\ cuceu_corina@yahoo.com (C.C.); graceshim1@gmail.com (G.S.); monika.frenzel@hotmail.com (M.F.); \\ mustafa.aljawhari@hotmail.fr (M.A.J.); williamhempel824@gmail.com (W.M.H.); \\ audelenain@yahoo.fr (A.L.); luc.morat@cea.fr (L.M.) \\ 2 IRIMAS, Institut de Recherche en Informatique, Mathématiques, Automatique et Signal, Université de \\ Haute-Alsace, 68093 Mulhouse, France; bruno.colicchio@uha.fr (B.C.); alain.dieterlen@uha.fr (A.D.) \\ 3 Department of Genetic, Groupe Hospitalier de la Région de Mulhouse Sud-Alsace, 68093 Mulhouse, France; \\ jeandidiere@ghrmsa.fr \\ 4 Institute of Biomedicine, University of Aarhus, DK-8000 Aarhus, Denmark; sjunker@biomed.au.dk \\ 5 Laboratory of Biochemistry B, Saint Louis Hospital, 75010 Paris, France; lfplassa@yahoo.fr \\ 6 Faculty of Automatic Control, Electronics and Computer Science, Silesian University of Techology, \\ 44-100 Gliwice, Poland; justyna.mika@polsl.pl (J.M.); Joanna.Polanska@polsl.pl (J.P.) \\ 7 Biological Effects Department, Centre for Radiation, Chemical and Environmental Hazards, Public Health \\ England, Didcot OX11 ORQ, UK; Sylwia.Kabacik@phe.gov.uk (S.K.); christophe.badie@phe.gov.uk (C.B.) \\ 8 Department of Radiation Oncology, Gustave Roussy Cancer Campus, 94805 Villejuif, France; \\ theogirinsky@me.com \\ 9 Department of Medicine, Gustave Roussy Cancer Campus, University Paris Saclay, 94805 Villejuif, France; \\ dr.pcarde@gmail.com \\ 10 Cell Environment DNA Damages R\&D Oncology Section, 75020 Paris, France \\ * Correspondence: radhia.mkacher@gmail.com or radhia.mkacher@cell-environment.com
}

Received: 7 May 2019; Accepted: 9 May 2019; Published: 30 May 2019

check for updates

The authors wish to make the following corrections to this paper [1]:

The previous 11th author, Grainne O'Brien, should be replaced with Sylwia Kabacik.

The "Authors Contributions" statement should be changed to:

Authors Contributions: Conceived and designed the experiments: R.M. Performed the experiments: C.C., R.M., M.F., L.M., F.P., S.K., G.S., and A.L. Analyzed the data: B.C., E.J., S.J., R.M., M.A.J., C.B., J.M., and P.C. Contributed reagents/materials/analysis tools: A.D., T.G., J.P., and P.C. Wrote the paper: R.M., W.M.H., P.C., E.J. and S.J.

The authors would like to apologize for any inconvenience caused to the readers by these changes. 


\section{Reference}

1. Cuceu, C.; Colicchio, B.; Jeandidier, E.; Junker, S.; Plassa, F.; Shim, G.; Mika, J.; Frenzel, M.; AL Jawhari, M.; Hempel, W.M.; et al. Independent Mechanisms Lead to Genomic Instability in Hodgkin Lymphoma: Microsatellite or Chromosomal Instability ${ }^{\dagger}$. Cancers 2018, 10, 233. [CrossRef] [PubMed] 